\title{
Base Composition and Translational Selection are Insufficient to Explain Codon Usage Bias in Plant Viruses
}

\author{
Daniel J. Cardinale, Kate DeRosa and Siobain Duffy * \\ Department of Ecology, Evolution, and Natural Resources, School of Environmental and Biological \\ Sciences, Rutgers, The State University of New Jersey, 14 College Farm Road, New Brunswick, NJ \\ 08901, USA; E-Mails: daniel.j.cardinale@gmail.com (D.C.), klderosa@eden.rutgers.edu (K.D.), \\ duffy@aesop.rutgers.edu (S.D.) \\ * Author to whom correspondence should be addressed; E-Mail: duffy@aesop.rutgers.edu (S.D.); \\ Tel.: +1-848-932-6299; Fax: +1-848-932-0312.
}

Received: 13 December 2012; in revised form: 9 January 2013 / Accepted: 11 January 2013/

Published: 15 January 2013

\begin{abstract}
Viral codon usage bias may be the product of a number of synergistic or antagonistic factors, including genomic nucleotide composition, translational selection, genomic architecture, and mutational or repair biases. Most studies of viral codon bias evaluate only the relative importance of genomic base composition and translational selection, ignoring other possible factors. We analyzed the codon preferences of ssRNA (luteoviruses and potyviruses) and ssDNA (geminiviruses) plant viruses that infect translationally distinct monocot and dicot hosts. We found that neither genomic base composition nor translational selection satisfactorily explains their codon usage biases. Furthermore, we observed a strong relationship between the codon preferences of viruses in the same family or genus, regardless of host or genomic nucleotide content. Our results suggest that analyzing codon bias as either due to base composition or translational selection is a false dichotomy that obscures the role of other factors. Constraints such as genomic architecture and secondary structure can and do influence codon usage in plant viruses, and likely in viruses of other hosts.
\end{abstract}

Keywords: synonymous codon usage; translational selection; genomic content; mutational bias 


\section{Introduction}

All organisms exhibit some degree of codon usage bias (CUB), the unequal usage of synonymous codons [1,2]. Codon bias may vary among genes of the same organism, which is associated with factors like asymmetrical mutation pressures or tissue-specific gene expression, but is relatively uniform within the most highly expressed genes [3-10]. CUB is often explained as the product of two potentially competing factors: genomic base composition and translational selection (Table 1). In the absence of other mutational and selective pressures, CUB should result from the genomic frequency of A, C, G and T being reflected in third positions. When CUB diverges from the null hypothesis of genomic nucleotide content, translational selection-selection for optimal speed and accuracy of translation - is routinely invoked. Translational selection should exert an influence on CUB because preferred codons tend to correlate with the most common tRNAs [11,12], allowing for faster, yet accurate, codon recognition and translation of highly expressed genes [13,14]. However, genomic composition and translational selection need not be acting antagonistically on CUB, and sequences can show CUB distinct from that predicted by either force.

When genomic base composition and known preferred codons correlate with observed CUB, both are potentially influencing CUB, and we cannot distinguish the relative strength of the forces. When observed CUB conflicts with the known preferred codons but adheres to genomic nucleotide content, the null hypothesis of overall base composition cannot be rejected, but translational selection can. Conversely, when preferred codons and observed CUB align, but CUB differs from that predicted by genomic base content, the null hypothesis can be rejected and translational selection deemed a more likely explanation. In the fourth case, neither overall base composition nor translational selection appears to be driving the observed CUB. This could be the result of direct conflict between the two forces yielding an intermediate state (i.e., the genome is enriched in adenine and suppresses cytosine, but the preferred codons tend to end in cytosine at the expense of NNA codons). Alternatively, another factor or factors must be influencing CUB. However, the vast majority of studies into the causes of CUB frame the question in terms of genomic base composition vs. translational selection, which precludes the consideration of additional important factors $[15,16]$. Considerations such as speciesspecific nmer promotion and suppression (e.g., GATC for methyl-directed mismatch repair in E.coli [17], CpG in mammalian genomes [18]) are known to affect CUB, but are rarely considered in analyses of codon usage. 
Table 1. Possible explanations for codon usage bias when the codon usage bias (CUB) of a gene of interest match or fail to match the genomic base composition and relative synonymous codon usage (RSCU) of a set of reference genes. For viruses, the comparison would be between the CUB of the viral genes and the CUB of their hosts.

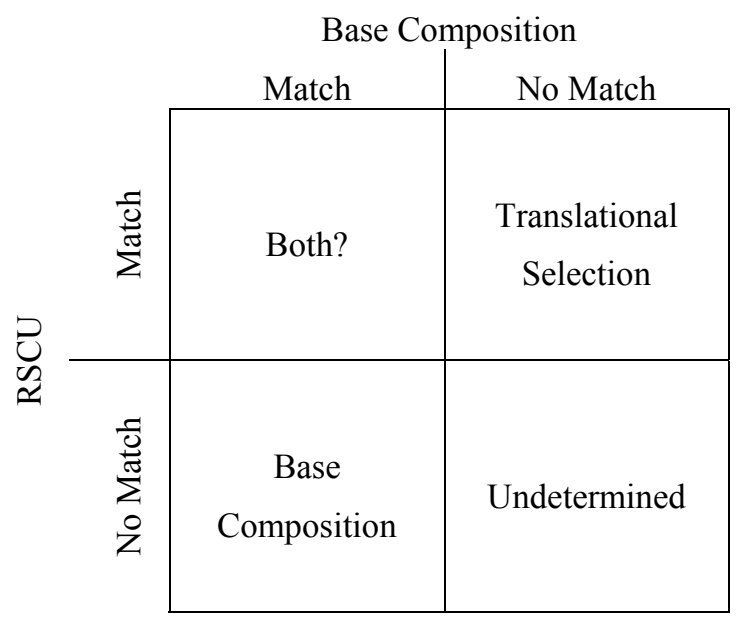

This framework can be applied to studies of viral codon bias. Viruses with well characterized hosts are ideal systems in which to explore the forces shaping CUB because their genomic biases can be calculated from their viral genomes, but the hosts' CUB reveal the translationally preferred codons. Viruses should experience translational selection to match the CUB of their hosts, as this should allow for faster translation of highly expressed viral genes, and consequently more rapid viral replication. It was recently documented that viruses with highly deoptimized CUB suffer a fitness cost [19]. However, surveys examining viral CUB have indicated that not all viruses are equally able to match their hosts' codon preferences, and that this may be correlated with viral genomic architecture [20]. For instance, we previously demonstrated that double-stranded (dsDNA) coliphages were significantly better matched to Escherichia coli's CUB than single-stranded (ssDNA) coliphages, because ssDNA phages had a preference for NNT codons, regardless of the hosts' preferred codon usage [21].

To further investigate the evolutionary forces shaping viral codon usage, we investigated patterns of CUB in plant viruses with distinct genomic architectures. Plant viruses offer a unique opportunity to examine CUB because plant virus families often include members that only infect monocot hosts, and others that only infect eudicot hosts - two distinct translational environments. Monocots tend to have GC biased genes (53-56\%), while eudicot genes generally have lower GC content (40-45\%)[22]. Monocots exclusively prefer G- and C-ending codons, while eudicots prefer a combination of G- and T-ending codons in their most highly expressed genes (Table 2). These divergent hosts allow the strength of translational selection pressures to be compared among related viruses.

We analyzed three large groups of arthropod-vectored plant viruses: the positive sense ssRNA genus Potyvirus and family Luteoviridae, and the ssDNA family Geminiviridae. Potyvirus and Geminiviridae contain a comparable number of species with at least 15 sequences available for analysis (22 and 24, respectively). There were fewer appropriate Luteoviridae for analysis (8), but similar to the Geminiviridae, monocot- and dicot- infecting luteoviruses are organized into separate genera. These three groups differ in their genomic architectures: the filamentous potyviruses have a linear $\sim 10 \mathrm{~kb}$ genome that is expressed as a polyprotein, luteoviruses contain a linear genome of 5.3- 
$5.7 \mathrm{~kb}$ that is translated from subgenomic RNAs, and geminiviruses have one or two circular, $\sim 2.7 \mathrm{~kb}$, ambisense genomic segments that are transcribed by host enzymes [23].

Unlike cellular organisms, which share related genes across extremely divergent clades, which can be used as the basis for phylogenies [24], very few functionally analogous viral genes are found in divergent taxa. We chose to examine the coat/capsid protein (CP) gene, a large ORF that is shared (though not homologous) among the three viral groups. While the CPs in some plant viruses serve the dual role of capsid and movement proteins[25], these factors only constrain amino acid usage, and should not impact synonymous codon usage. Similarly, the CPs of vectored viruses are under more strict selection against amino acid substitutions than those of non-vectored viruses [26], but as these arthropod-borne viruses are not expressing genes in the vector, it should not affect their codon bias. Therefore, analysis of CP genes best facilitates comparisons of results between the ssDNA and ssRNA viruses in this study.

Additional analyses were required in Geminiviridae. The potyvirus CP and the luteovirus CP are each considered monophyletic; the monocot- and dicot- infecting viral sequences within each group once shared a common ancestral sequence. The monophyly of the CP in the Geminiviridae is assumed based on its unusual capsid shape [27], but the protein sequence of the ORF is highly divergent between begomoviruses and mastreviruses. Consequently, we also analyzed the CUB of the replication-associated gene (Rep), which is encoded in the complementary sense, for the geminiviruses. There is strong phylogenetic evidence for their Reps to be descended from a common ancestor [28,29]. While their CPs may be useful for comparisons to the RNA viruses, comparisons of the Rep CUB within Geminiviridae may be more appropriate, and comparable to analyses of the homologous CPs within each RNA virus family [27,30].

We compared the relative synonymous codon usage (RSCU [4]) of monocot- and eudicot-infecting members of each group to their hosts, to each other, and to viral genomic nucleotide composition to assess the relative importance of host codon preferences in viral CUB. Our results were surprisingly varied for viruses infecting common hosts, and demonstrate that pressures beyond base composition and translational selection affect CUB in ssDNA and ssRNA plant viruses.

\section{Results}

\subsection{Monocots and Eudicots exhibit divergent $C U B$}

Plant codon preferences varied considerably between monocots and eudicots. The monocots analyzed exclusively preferred C- and G-ending codons in their highly expressed genes; fifteen of their overrepresented codons were C-ending, while the remaining five were G-ending (Table 2). These patterns were consistent with the codon preferences of all monocot genes [22]. Conversely, eudicots preferred a combination of NNT (six) and NNG (four) codons in their most highly expressed genes, in addition to two NNC codons (Table 2), which also agreed with their overall codon preferences. In all cases where plants preferred an NNG codon, no pyrimidines were possible in the third position (they were two-fold redundant amino acids, or the two-fold redundant portion of six-fold redundant amino acid codons). The RSCU of the highly expressed genes in the monocots and eudicots [22] were strongly correlated for A or T-ending codons $(\mathrm{r}=0.93)$, and for $\mathrm{G}$ or $\mathrm{C}$-ending codons 
$(\mathrm{r}=0.82$, Figure 1). However, best-fit lines for the two groups of codons both differ from a line with a slope of 1 through the origin, indicative of divergent codon preferences. Consequently, monocots and eudicots represent distinct translational environments, and should exert dissimilar translational selection pressures on the CUB of their respective viruses.

Table 2. Preferred codons in monocots and eudicots. Preferred codons are those with relative synonymous codon usage (RSCU) values that significantly exceed those of all synonymous codons ( $\mathrm{p}<0.05$, Bonferroni-corrected 2-tailed t-tests).

\begin{tabular}{|c|c|c|c|c|c|c|}
\hline & & onoc & & & udicot & \\
\hline & tac & aac & $\operatorname{ccc}$ & tac & $\mathrm{aac}$ & \\
\hline & ctc & acc & gac & & & \\
\hline $\mathrm{NNC}$ & atc & $\mathrm{gcc}$ & $\operatorname{tgc}$ & & & \\
\hline & tcc & tac & $\operatorname{cgc}$ & & & \\
\hline & $\mathrm{agc}$ & $\mathrm{cac}$ & $\mathrm{ggc}$ & & & \\
\hline $\mathrm{NNG}$ & $\mathrm{ttg}$ & aag & cag & $\mathrm{ttg}$ & aag & cag \\
\hline & gag & agg & & gag & & \\
\hline & & & & $\mathrm{ctt}$ & tct & gct \\
\hline N & & & & $\mathrm{gtt}$ & gat & cgt \\
\hline
\end{tabular}


Figure 1. Relative synonymous codon usage (RSCU) correlation between monocot and eudicot highly expressed genes. Triangles represent $\mathrm{A} / \mathrm{T}$, squares are $\mathrm{C} / \mathrm{G}$, open symbols are pyrimidines, closed are purines. Solid line is best fit for A/T-ending codons $(\mathrm{r}=0.93)$, dashed is for $\mathrm{G} / \mathrm{C}$-ending $(\mathrm{r}=0.82)$. The grey line has a slope of 1 through the origin.

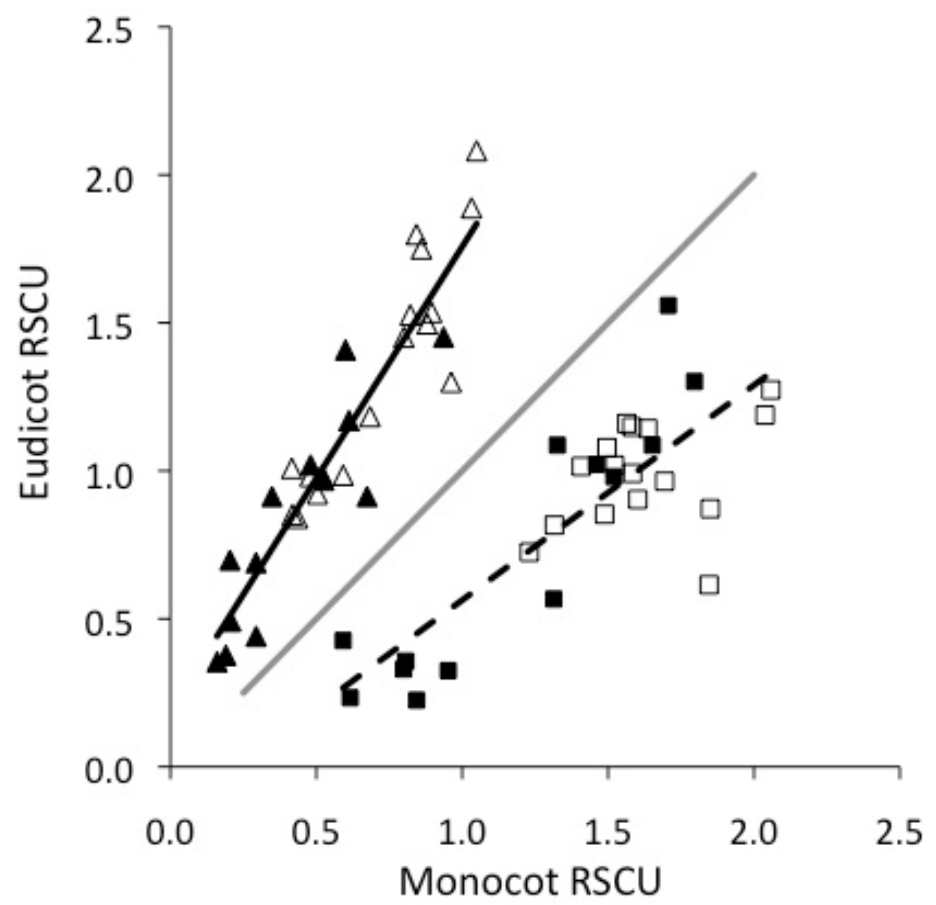

\subsection{Base composition does not explain most CUB in plant viruses}

The potyviruses showed a consistent pattern of elevated adenine in their genomes, regardless of host, and also contained correspondingly lower levels of cytosine and guanine. The third position nucleotide content in $\mathrm{CP}$ genes differed significantly from that of the overall genome in every potyvirus we examined (chi-square tests, $\mathrm{p}<0.05$ ). Luteoviruses showed consistent genomic base composition, having slightly elevated genomic adenine content, and relatively equitable use of cytosine, guanine, and thymine. Third position base frequencies were also consistent regardless of host, but differed significantly from genomic nucleotide composition in most luteoviruses. Third position base usage in two of the four eudicot-infecting luteoviruses did not differ significantly from genomic base content (chi-square tests, $\mathrm{p}>0.1$ ). In the two remaining eudicot-infecting, and all four monocot-infecting luteoviruses, third positions diverged significantly from the genomes (chi-square tests, $\mathrm{p}<0.05$ ). These findings indicate that genomic base composition is a poor predictor of CUB in luteoviruses and potyviruses.

Average third position base composition of $\mathrm{CP}$ genes in begomoviruses (dicot-infecting geminiviruses) and mastreviruses (monocot-infecting geminiviruses) also varied greatly from their respective genomic nucleotide contents (18 begomoviruses and 4 mastreviruses, chi-square tests, $\mathrm{p}<0.001)$. Base composition of synonymous sites in the Rep genes of all begomoviruses $(\mathrm{n}=14)$ and two out of three mastreviruses also diverged substantially from overall genomic nucleotide content (chi-square tests, $\mathrm{p}<0.05$ ). As is the case for the ssRNA viruses, these results strongly suggest that genomic base composition does not drive CUB in geminiviruses. 


\subsection{RNA virus $C U B$ is independent of host use}

All potyviruses had somewhat similar codon preferences, independent of host: monocot- and eudicot-infecting potyviruses both generally preferred A- and T-ending codons (Table 3). Luteoviruses, both monocot- and eudicot-infecting, exhibited preferences for fewer codons overall, but tended to favor NNC codons. Despite this overall similarity they shared only two preferred codons (Table 3).

Table 3. Preferred codons in luteoviruses and potyviruses infecting monocot and eudicot hosts. Preferred codons are those with relative synonymous codon usage (RSCU) values that significantly exceed those of all other synonymous codons $(p<0.05$, Bonferroni-corrected 2-tailed t-tests).

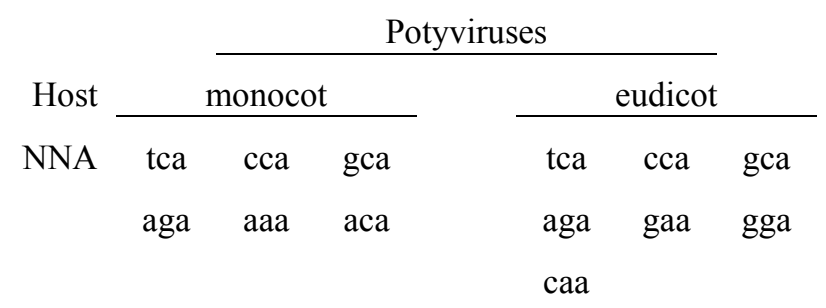

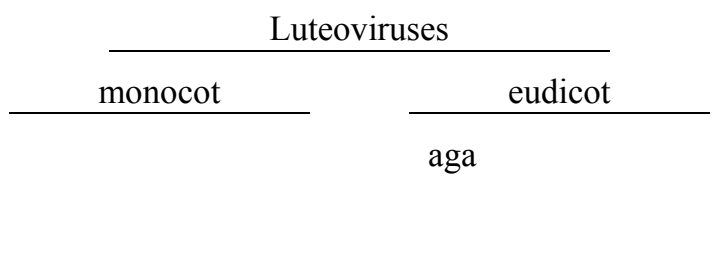

$\begin{array}{lllllll}\text { NNC } & \text { tgc } & \text { cac } & & & \\ & & & & & \\ & & & & & & \\ & & & & \text { tat aat gat }\end{array}$

NNG $\quad \operatorname{tg} \quad$ agg

Eudicot-infecting potyvirus RSCU was moderately correlated with eudicot RSCU ( $\mathrm{r}=0.55)$, and monocot-infecting RSCU was actually weakly anti-correlated with monocot RSCU ( $\mathrm{r}=-0.29$, Figure 2a). Surprisingly, monocot-infecting potyvirus RSCU correlated with eudicot RSCU nearly as well as eudicot-infecting potyviruses $(\mathrm{r}=0.46)$. Monocot- and eudicot-infecting luteovirus RSCU weakly correlated with that of their hosts $(r=0.29$ and 0.16 , respectively, Figure $2 b)$. 
Figure 2. Correlation between host and virus coat/capsid protein $(\mathrm{CP})$ relative synonymous codon usage (RSCU) for monocot-infecting (red) and eudicot-infecting (blue) (a) potyviruses and (b) luteoviruses.

(a)

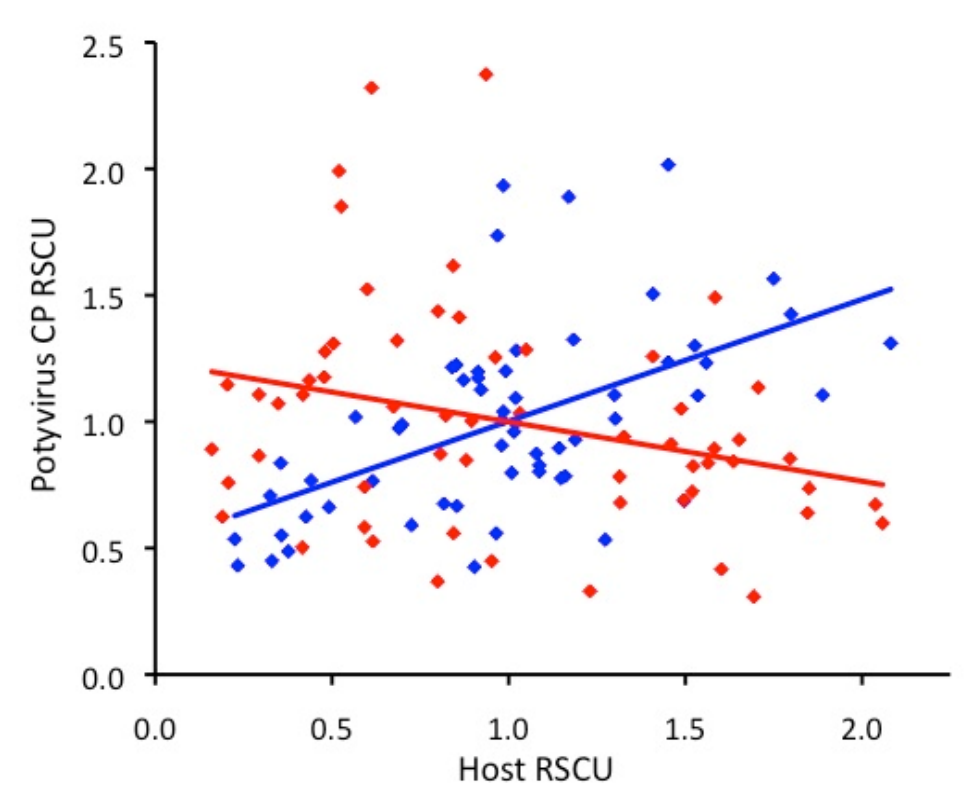

(b)

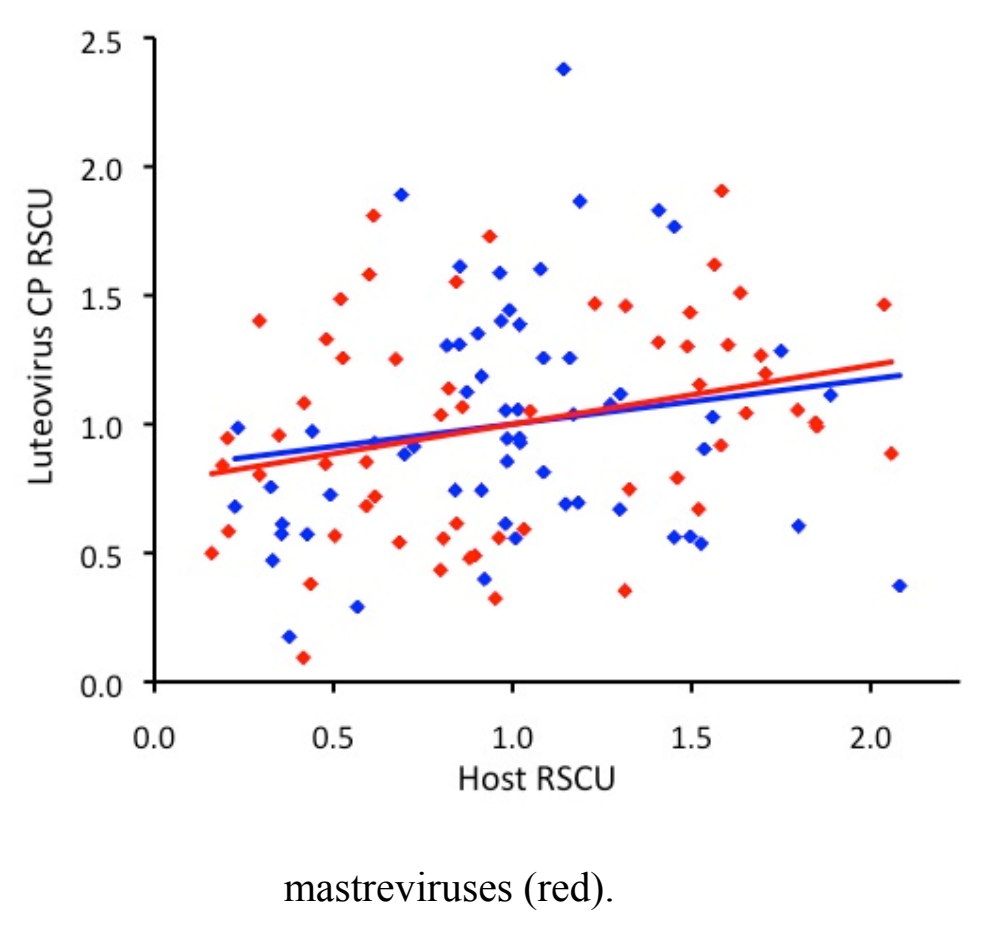

2.4. ssDNA virus $R S C U$ does not indicate strong translational selection

ssDNA dicot-infecting begomovirus CP genes exhibited a strong preference for NNT codons, while begomovirus Rep sequences strongly favored NNA codons (Table 4). In the monocot-infecting 
mastreviruses, CP genes preferentially used C- and G-ending codons, but the Rep sequences did not exhibit a specific preference; overrepresented codons ended in all four bases (Table 4). Begomovirus genomes are ambisense; genes are encoded in the coding and complimentary sense [31]. The coding sequence of the Rep gene is complimented on the virion strand. As a consequence, third positions in this gene are present as the first base of anti-codons in the single-stranded viral genome. Therefore, these findings indicate begomovirus genomes are enriched for thymine at synonymous sites in both the CP ORF (with T-ending codons) and Rep ORF (with T-beginning anticodons).

Table 4. Preferred codons in mastrevirus and begomovirus Rep and CP genes. Preferred codons are those with relative synonymous codon usage (RSCU) values that significantly exceed those of all other synonymous codons $(p<0.05$, Bonferroni-corrected 2-tailed t-tests).

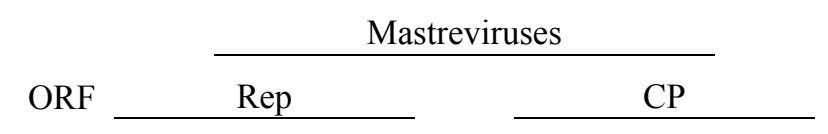

NNA aaa

$\mathrm{NNC}$ tac

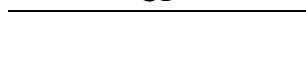

ttc gec gac

agt

cat

aat

$\begin{array}{lll}\text { cat } & \text { cgt } & \text { aat } \\ \text { gat } & \text { act } & \text { tgt } \\ \text { att } & \text { ggt } & \text { gtt } \\ \text { tat } & & \end{array}$

$\operatorname{ttg}$

aag cag ctg
Begomoviruses

\begin{tabular}{lllll} 
& Rep & & & CP \\
\hline aaa & caa & gga & & \\
aca & cca & gaa & \\
aga & & &
\end{tabular}

ttc $\quad$ ctc $\operatorname{tgc}$

ttc $\quad \operatorname{ccc}$

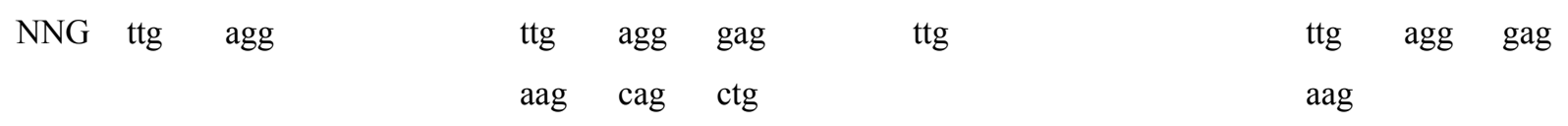

In both begomoviruses and mastreviruses, the RSCU of their CP genes was moderately correlated to the RSCU of the highly expressed genes of their respective hosts ( $\mathrm{r}=0.69$ for begomoviruses, 0.53 for mastreviruses, Figure 3a). However, the Rep gene was not well correlated to their host RSCU in either begomoviruses $(\mathrm{r}=0.38)$ or mastreviruses $(\mathrm{r}=0.06$, Figure $3 \mathrm{~b})$. Mastrevirus Rep RSCU actually matched that of eudicots better than the begomovirus Rep RSCU ( $\mathrm{r}=0.71$ ). 
Figure 3. Correlation between relative synonymous codon usage (RSCU) of (a) geminivirus $\mathrm{CP}$ and (b) geminivirus Rep and host RSCU for eudicot-infecting begomoviruses (blue) and monocot-infecting mastreviruses (red).

(a)

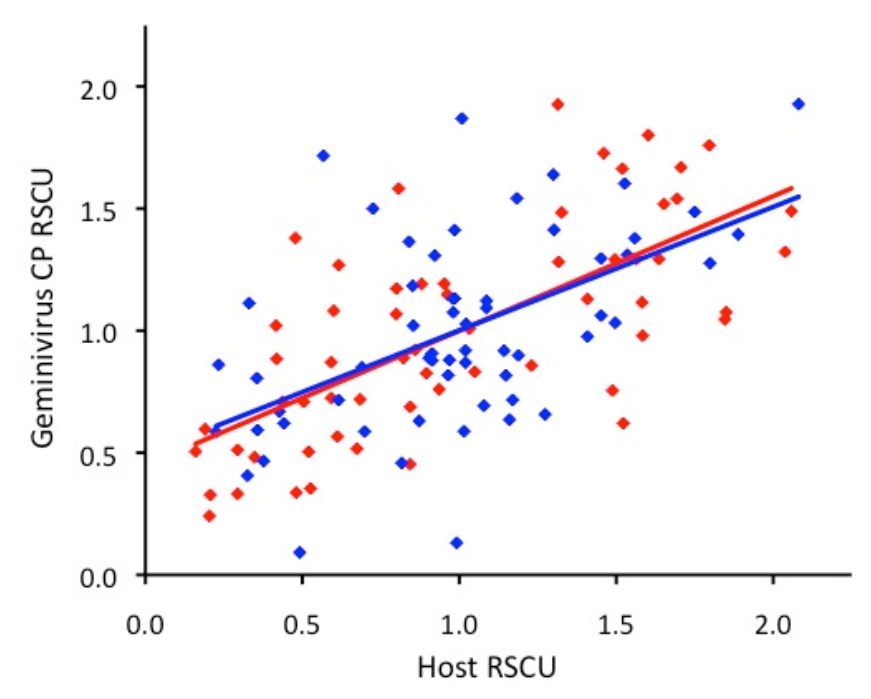

(b)

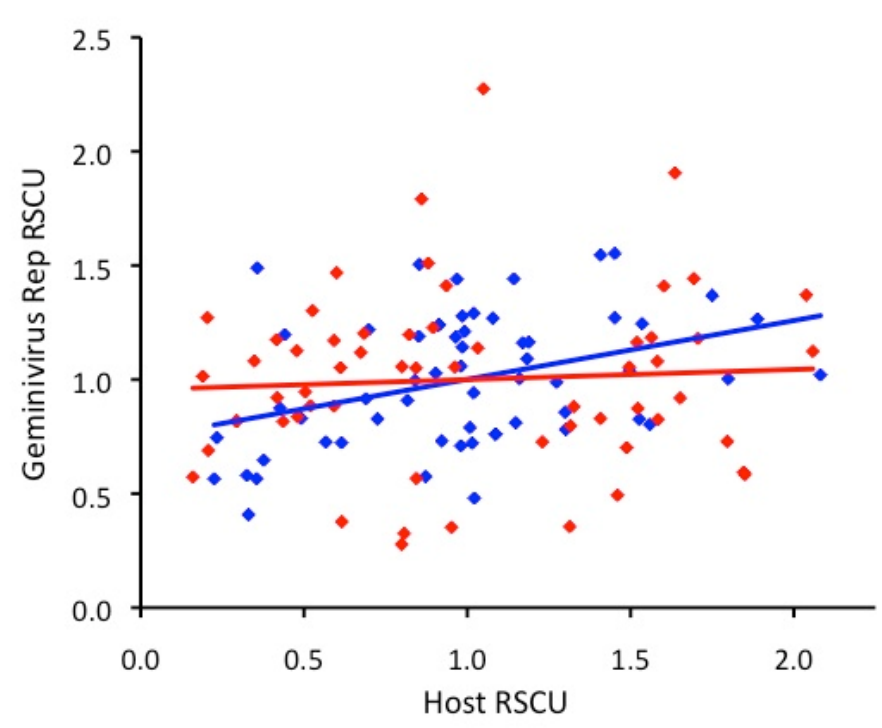

The conservation of codon usage between monocot- and eudicot- infecting viruses within each group varied significantly. Potyvirus RSCUs were strongly correlated to each other $(\mathrm{r}=0.90)$, despite their hosts having divergent preferences (Figure 4a). Similar to the potyviruses, the two luteovirus groups exhibited a moderate correlation to each other $(\mathrm{r}=0.66$, Figure $4 \mathrm{a})$. Begomovirus and mastrevirus RSCUs were uncorrelated in the CP ORF ( $\mathrm{r}=0.16)$, but moderately correlated in the Rep ORF ( $r=0.51$, Figure $4 b)$. 
Figure 4. Correlation of relative synonymous codon usage (RSCU) between (a) monocot and eudicot-infecting potyvirus CPs (orange), luteovirus CPs (green) and (b) begomo- and mastrevirus CPs (aqua) and begomo- and mastrevirus Reps (purple).

(a)

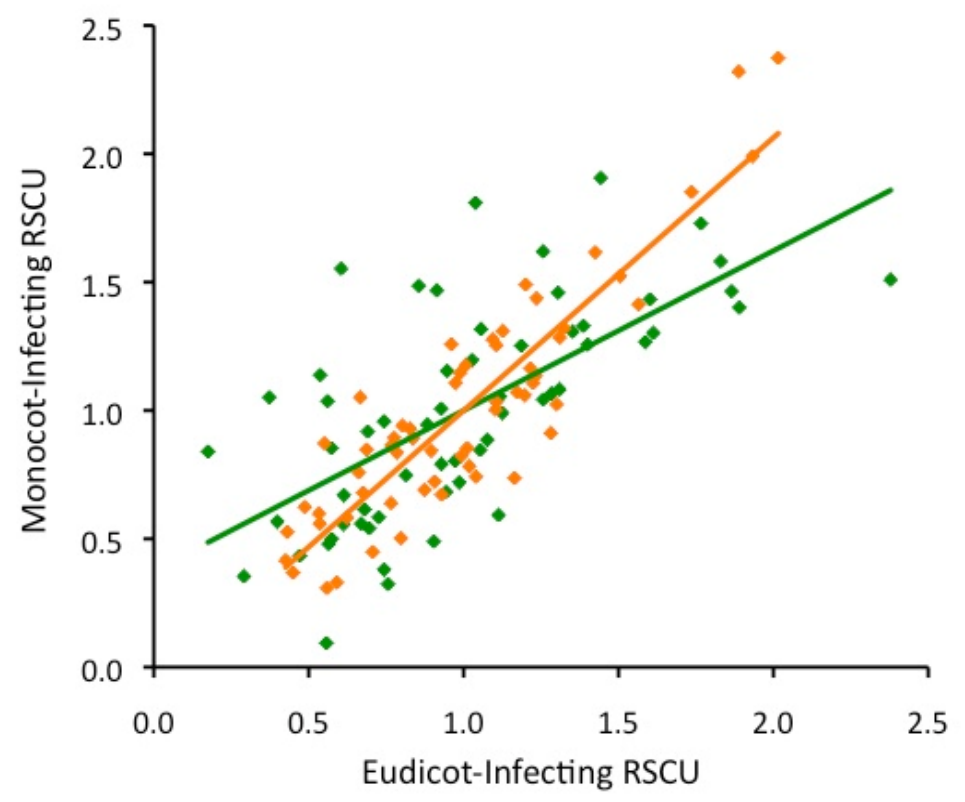

(b)

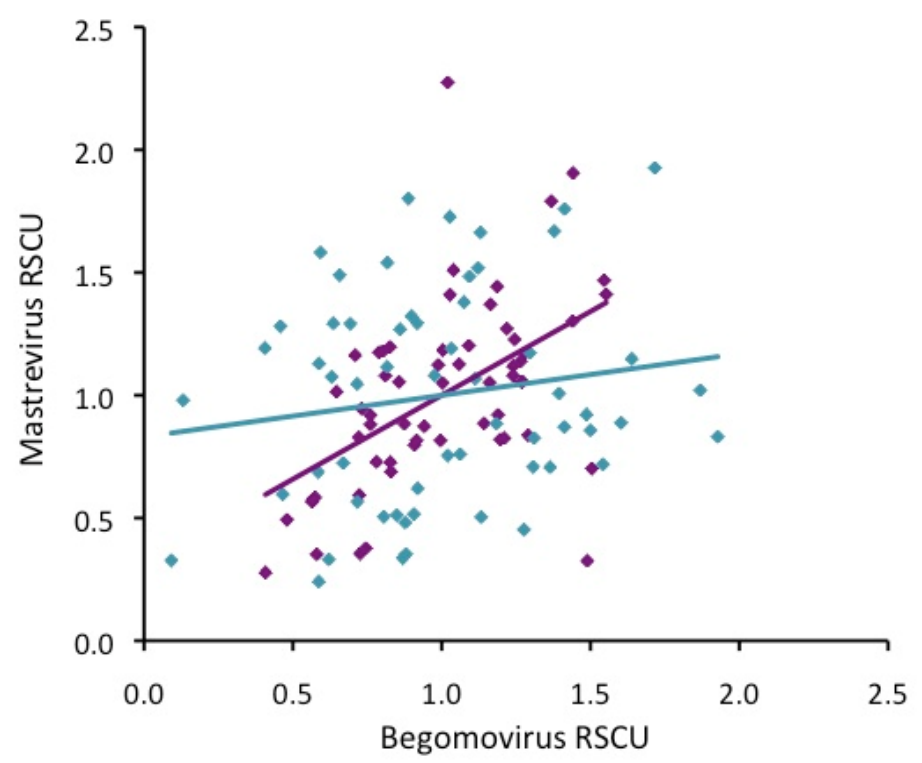




\section{Discussion}

\subsection{Neither base composition nor translational selection explains our results}

The null hypothesis, that synonymous codon usage is purely a function of the nucleotide base frequencies in the viral genome, was insufficient to explain codon preferences in all of the groups we examined. The discord between genomic frequencies and third position frequencies did not often manifest as more equitable nucleotide use in the third position; instead, codon use was more biased than genomic nucleotide frequencies in several cases (eudicot-infecting potyviruses, begomovirus CP). Similarly, the alternative hypothesis of translational selection failed to explain much of the plant virus CUB. Dicot-infecting potyviruses and geminivirus CP CUB were moderately correlated with their host CUB, but we did not find as high a correlation as in phage [21] or human RNA viruses [20].

The most common methods of assessing the relationship between genomic base composition and CUB involve using $\mathrm{GC}_{3}$, the $\mathrm{GC}$ content of the third codon position, as a measure of codon bias. $\mathrm{GC}_{3}$ is then compared to $\mathrm{GC}_{1,2}$, the $\mathrm{GC}$ content of the first and second codon positions, or ENC, the effective number of codons [32,33]. Both measures vary along predictable lines or curves when base composition drives CUB. By these measures, CUB in many viruses is strongly affected by overall base composition [33-35]. However, metrics that group AT and GC are unable to account for the over- or underrepresentation of a specific nucleotide in the third position at the expense of its complement, which is of special concern when analyzing CUB in single-stranded viruses. In the begomoviruses we analyzed, the average $\mathrm{GC}_{3}$ is approximately equal in the CP, Rep, and whole genome, but the CP (and genome) favor guanine while cytosine is overrepresented in the Rep. Consequently, our methods require a higher degree of agreement between the overall genomic base content and gene third position for base composition to be considered a plausible explanation for CUB.

Furthermore, many studies of viral CUB do not explicitly evaluate other factors that can drive codon bias. Rather, the null hypothesis is tested - does CUB follow the predicted relationship between $\mathrm{GC}_{3}$ and $\mathrm{GC}_{1,2}$ or $\mathrm{ENC}$ - and if not rejected, the effects of translational selection and other possible factors are not subsequently analyzed [15,16,34,36]. Other studies attribute the rejection to translational selection, but fail to consider additional factors [37-39].

\subsection{Possible alternative explanation for CUB in plant viruses}

Genes of propagative arthropod-vectored viruses (those that replicate within their vectors) should be under dual selective pressures to maximize replication speed within their plant hosts and their vectors. Consequently, vector codon preferences could influence codon bias in these viruses. However, the potyviruses and luteoviruses are nonpropagative [40], and while the evidence is more ambiguous in geminiviruses, they are generally considered nonpropagative as well [41,42], so translational selection is not acting on these viral genomes in their respective vectors. There is evidence of heightened purifying selection on capsid structure in vectored RNA viruses due to specific interactions between $\mathrm{CP}$ and vector [26], but in nonpropagative viruses, this pressure is independent of translation kinetics, instead acting solely on amino acid sequence. Therefore, translational selection within arthropod vectors cannot explain the observed CUB. 
As potyviruses have high mutation rates [43], have diverged over at least thousands of years [44], and the different species analyzed were at least $25 \%$ divergent by nucleotide [23], it is impossible that the common synonymous codon usage we observed is an accident of recent fixation. It is similarly unlikely that a recent host-shift from eudicots (to which potyvirus CUB is better matched) brought recently diverged potyviruses into monocots [44]. Luteoviruses [45] and geminiviruses [46-48] evolve at similarly high speeds, so it is unlikely that these correlations are due to accidental historical contingency. It would further be expected that third positions would be saturated after thousands, if not millions of years of divergence [49]. Given these factors, it is most likely that the correlation among monocot- and eudicot-infecting members of each group is due to a similar set of pressures affecting CUB of each group as a whole.

One possible factor that may influence codon bias in ssRNA viruses is selective constraints on secondary structure. ssRNA viral genomes often contain complex secondary structures that are important for replication or gene expression [50]. Disruption of these structures can inhibit one or both processes, reducing viral fitness. Substitutions are often observed in pairs: an initial mutation and a compensatory mutation that restores base pairing across stems in stem-loop structures, for instance [51]. These factors should manifest as more constrained codon usage at specific sites, though the effects on overall codon usage are ambiguous.

The begomovirus CP, which strongly preferred NNT codons, aligned well with the preference of their eudicot hosts for T-ending codons and correlated strongly with host RSCU. Despite the significant differences between third position and genomic base content, these results also indicate the potential importance of the thymine enrichment of high-AT begomovirus genomes. Therefore, it is tempting to explain these data as the result of the combination of compositional constraints and strong translational selection, even if third position base use significantly differed from that of the entire genome. Conversely, begomovirus Rep sequences have different preferences and demand a different explanation. CUB is not explained by base composition, but the prevalence of A-ending codons and the weak correlation ( $r=0.37$, Figure $4 \mathrm{~b})$ between host and virus RSCU suggests weak translational selection. It is unlikely that these two genes are subject to such divergent pressures that they would exhibit such inverse biases, as CUB tends to be similar within species [52]. However, neither base composition nor translational selection is sufficient to explain begomovirus CUB.

Similarly, CUB in the mastreviruses is not easily explained. In particular, the Rep has no welldefined codon preferences, which is not predicted by the genomic nucleotide composition, translational selection for their hosts' CUB, nor an antagonistic relationship between the two. Consequently, these two factors alone are not sufficient to explain mastrevirus CUB.

The ssDNA architecture of geminiviruses provides a possible explanation for their CUB. ssDNA is prone to rapid cytosine deamination to uracil [53], and this process may explain the preference for T-ending codons in ssDNA phages, even in hosts with low AT\% [21]. If this process also affects eukaryotic ssDNA viruses, we would expect a very different CUB profile compared to that which is determined by only base content and translational selection. Specifically, strong translational selection predicts uniform codon usage in both CP and Rep, but a strong, biased mutational pressure predicts the begomovirus preference for A-ending codons in the Rep sequences, given that they are encoded in the negative sense. $\mathrm{C} \rightarrow \mathrm{T}$ transitions may be tolerated only at synonymous sites, resulting in an overabundance of thymine in the genomic sequence, and a corresponding preference for adenine in the 
Rep coding sequence. When viewed as they are encoded in the genome, begomovirus CP and Rep nucleotide preferences at synonymous sites are remarkably consistent: both strongly prefer T-ending codons/T-beginning anticodons, suggesting that this biased mutational pressure may contribute to geminivirus CUB. A recent study of the ssDNA porcine circovirus also shows a preference for T-ending codons that differs from the codon preferences of their swine hosts, and is not due to genomic composition [54].

We believe it is very likely that a biased $\mathrm{C} \rightarrow \mathrm{T}$ mutational pressure affects eukaryotic ssDNA viruses. Eudicot-infecting begomoviruses are known to exhibit a long-term $\mathrm{C} \rightarrow \mathrm{T}$ substitution bias [46,47]. Additionally, ssDNA phages typically exhibit little secondary structure [55-60], and while very limited degrees have been documented in eukaryotic ssDNA viruses [61], it has not been observed in the ORFs we examined here. Consequently, these genomes are unconstrained by structural constraints at synonymous sites, and, because unpaired DNA is 100 times more susceptible to oxidative cytosine deamination to uracil than dsDNA [53], highly vulnerable to $\mathrm{C} \rightarrow \mathrm{T}$ transition. These oxidative deaminations are common in cellular genomes, but are efficiently repaired [62], while such changes in ssDNA viruses might simply go unrepaired [63]. Alternatively, host cytidine deaminases could be increasing viral thymine content by enzymatically deaminating cytosines. These enzymes are an innate mammalian anti-viral defense, and are active against both viral RNA and ssDNA [64]. Regardless of the exact mechanism, the evidence points to a biased mutational pressure at cytosines contributing to begomovirus evolution.

Mastreviruses present a contrary case: whether or not they experience this potential thymineenriching factor, their CUB remains unlikely in the absence of additional drivers. Mastreviruses may not experience the same mutational pressure from deamination, may have developed ways to compensate for it, or monocot hosts may interact with ssDNA genomes differently than eudicots. Neither their CP nor Rep sequences carry the signature of rapid deamination, and their CP CUB strongly adheres to host preferences, indicating the primacy of translational selection over base composition and other potential factors. Furthermore, an examination of maize streak virus revealed no evidence of the long-term $\mathrm{C} \rightarrow \mathrm{T}$ substitution bias evident in begomoviruses [48]. Finally, unlike most organisms that have been studied, MSV exhibits high degrees of variance in CUB between different genes, and the reasons for this variation are unclear [33]. A single recently discovered eudicot-infecting mastrevirus sequence [65] exhibited codon usage preferences that differ from monocots, eudicots, and the other viruses we examined. Additional analysis is required to more precisely determine the forces affecting CUB in mastreviruses.

\section{Methods}

\subsection{Host codon usage bias}

Codon preferences in highly expressed genes for five monocot and six eudicot plants were determined using the RSCU data from Wang and Roossinck [22]. Average monocot and eudicot RSCU was calculated for each codon, and preferred codons were defined by Bonferroni-corrected twotailed t-tests (Microsoft Excel) of average RSCU for synonymous codons. Each set of redundant codons was analyzed individually; no comparisons were made between non-redundant codons. 
For these analyses, codons for the six-fold degenerate amino acids (L, R, S) were divided into two-fold and four-fold redundant groups, for which RSCU values were calculated independently. This was done because the two groups of codons for these amino acids differ at non-synonymous sites and are consequently recognized by different groups of tRNA species, making it inappropriate to treat them as a single set of redundant codons.

\subsection{Plant virus datasets}

All available complete CP sequences of luteoviruses and potyviruses were collected from GenBank between March and May of 2012. Only species with 15 or more full CP gene sequences were analyzed, and sequences with ambiguous nucleotides were excluded. Complete CP and complete Rep gene sequences of Geminiviruses (monocot-infecting mastreviruses and dicot-infecting begomoviruses) were downloaded from GenBank between January and April of 2012. As with the ssRNA viruses, only species with at least 15 full gene sequences were analyzed, and sequences with ambiguous nucleotides were excluded. Consequently, some geminivirus species could be included in one of our analyses (CP analysis) but not the other (Rep analysis). In total, we analyzed 1285 geminivirus Rep gene sequences, 1481 geminivirus, 1210 potyvirus, and 315 luteovirus CP gene sequences (Supplemental File 1).

\subsection{Base composition as a null hypothesis}

All sequences were formatted for analysis using ReadSeq (http://wwwbimas.cit.nih.gov/molbio/readseq). CAICal [66] was used to calculate the viral base composition. Reference sequences for each viral species were collected from GenBank on June 12, 2012. Sequences were formatted with ReadSeq, and CAICal was used to determine overall and site-specific base composition for each sequence. Observed third position nucleotide counts were averaged for each species. Expected third position nucleotide counts were computed for each gene/species combination we analyzed based on the genomic nucleotide frequencies of the species' reference genome and the length of the ORF in the reference genome. Chi-square tests were used to evaluate the differences between these observed and expected counts, with three degrees of freedom (MS Excel). In total, sixty-seven chi-square tests were carried out: one on the $\mathrm{CP}$ gene of each potyvirus, luteovirus, and geminivirus we examined, and one on the Rep gene of each geminivirus we analyzed.

\subsection{Plant virus codon usage biases}

Viral RSCU calculations were as for the plant hosts. RSCU values were calculated for each sequence in each viral genus (in the case of the potyviruses, monocot-infecting and dicot-infecting). Then, mean RSCU values were calculated for each viral genus/group. Preferred codons were again defined by Bonferroni-corrected two-tailed t-tests of average RSCU for synonymous codons, and determined separately for monocot- and dicot-infecting members of each viral group. 


\subsection{Comparison with host CUB}

Average RSCU values for the monocot- and eudicot-infecting viruses of each viral group were compared to those of their respective hosts to determine the correlation between host and viral CUB. Average RSCU of monocot-infecting and dicot-infecting viruses within each group were also compared to each other to determine if the correlation among related viruses was stronger than the correlation to their respective hosts. RSCU between two groups was classified as uncorrelated $(\mathrm{r}<0.50)$, moderately correlated $(0.50 \leq \mathrm{r}<0.70)$, or strongly correlated $(\mathrm{r} \geq 0.70)$. Translational selection was rejected when host and virus RSCU were uncorrelated, but considered in cases of moderate or strong correlation.

\section{Conclusions}

Codon usage bias is most often presented as the result of two competing forces: translational selection and genomic base composition. The methods most often used to evaluate it are sometimes sufficient to distinguish one of these factors from the other. However, in situations where neither factor appears significant, the available methods are of little use. Viral genomic nucleotide composition does not appear to be driving CUB in plant viruses, but there is only weak evidence of translational selection influencing CUB. Present methods are unable to explain plant virus CUB. Therefore, new ways of analyzing CUB and evaluating its likely determinants are required to more accurately parse the large amount of genomic data now available.

\section{Acknowledgments}

SD was funded by Rutgers School of Environmental and Biological Sciences and the New Jersey Agricultural Experiment Station.

\section{Conflict of Interest}

The authors declare no conflict of interest.

\section{References}

1. Shields, D.C.; Sharp, P.M.; Higgins, D.G.; Wright, F. "Silent" sites in drosophila genes are not neutral: Evidence of selection among synonymous codons. Mol. Biol. Evol. 1988, 5, 704-716.

2. Aota, S.; Ikemura, T. Diversity in $\mathrm{g}+\mathrm{c}$ content at the third position of codons in vertebrate genes and its cause. Nucleic Acids Res. 1986, 14, 6345.

3. Bennetzen, J.L.; Hall, B.D. Codon selection in yeast. J. Biol. Chem. 1982, 257, 3026-3031.

4. Sharp, P.M.; Li, W.-H. An evolutionary perspective on synonymous codon usage in unicellular organisms. J. Mol. Evol. 1986, 24, 28-38.

5. Karlin, S.; Mrázek, J.; Campbell, a.M. Codon usages in different gene classes of the escherichia coli genome. Mol. Microbiol. 1998, 29, 1341-1355.

6. Hershberg, R.; Petrov, D.A. Selection on codon bias. Annu. Rev. Genet. 2008, 42, 287-299. 
7. Hiraoka, Y.; Kawamata, K.; Haraguchi, T.; Chikashige, Y. Codon usage bias is correlated with gene expression levels in the fission yeast schizosaccharomyces pombe. Genes Cells 2009, 14, 499-509.

8. Bailly-Bechet, M.; Danchin, A.; Iqbal, M.; Marsili, M.; Vergassola, M. Codon usage domains over bacterial chromosomes. PLoS Comput. Biol. 2006, 2, e37.

9. Lobry, J.R.; Sueoka, N. Asymmetric directional mutation pressures in bacteria. Genome Biol. 2002, 3 .

10. Camiolo, S.; Farina, L.; Porceddu, A. The relation of codon bias to tissue-specific gene expression in arabidopsis thaliana. Genetics 2012, 192, 641-649.

11. Ikemura, T. Correlation between the abundance of escherichia coli transfer rnas and the occurrence of the respective codons in its protein genes: A proposal for a synonymous codon choice that is optimal for the e. Coli translational system. J. Mol. Biol. 1981, 151, 389-409.

12. Ikemura, T. Correlation between the abundance of yeast transfer rnas and the occurrence of the respective codons in protein genes: Differences in synonymous codon choice patterns of yeast and escherichia coli with reference to the abundance of isoaccepting transfer rnas. J. Mol. Biol. 1982, 158, 573-597.

13. Curran, J.F.; Yarus, M. Rates of aminoacyl-trna selection at 29 sense codons in vivo. J. Mol. Biol. 1989, 209, 65-77.

14. Pedersen, S. Escherichia coli ribosomes translate in vivo with variable rate. The EMBO Journal 1984, 3, 2895-2898.

15. Cai, M.-S.; Cheng, A.-C.; Wang, M.-S.; Zhao, L.-C.; Zhu, D.-K.; Luo, Q.-H.; Liu, F.; Chen, X.Y. Characterization of synonymous codon usage bias in the duck plague virus ul35 gene. Intervirology 2009, 52, 266-278.

16. Jia, R.; Cheng, A.; Wang, M.; Xin, H.; Guo, Y.; Zhu, D.; Qi, X.; Zhao, L.; Ge, H.; Chen, X. Analysis of synonymous codon usage in the ul24 gene of duck enteritis virus. Virus Genes 2008, $38,96-103$.

17. Au, K.G.; Welsh, K.; Modrich, P. Initiation of methyl-directed mismatch repair. J. Biol. Chem. 1992, 267, 12142-12148.

18. Krieg, A.M.; Yi, A.-K.; Matson, S.; Waldschmidt, T.J.; Bishop, G.A.; Teasdale, R.; Koretzky, G.A.; Klinman, D.M. Cpg motifs in bacterial DNA trigger direct b-cell activation. Nature 1995, $374,546-549$.

19. Bull, J.J.; Molineux, I.J.; Wilke, C.O. Slow fitness recovery in a codon-modified viral genome. Mol. Biol. Evol. 2012, 29, 2997-3004.

20. Jenkins, G.M.; Holmes, E.C. The extent of codon usage bias in human RNA viruses and its evolutionary origin. Virus Res. 2003, 92, 1-7.

21. Cardinale, D.J.; Duffy, S. Single-stranded genomic architecture constrains optimal codon usage. Bacteriophage 2011, 1, 219-224.

22. Wang, L.; Roossinck, M.J. Comparative analysis of expressed sequences reveals a conserved pattern of optimal codon usage in plants. Plant Mol. Biol. 2006, 61, 699-710.

23. King, A.M.Q.; Adams, M.J.; Carstens, E.B.; Lefkowitz, E.J. Virus taxonomy: Classification and nomenclature of viruses: Ninth report of the international committee on the taxonomy of viruses. Elsevier Academic press: San Diego, CA, USA, 2011. 
24. Woese, C.R.; Kandler, O.; Wheelis, M.L. Towards a natural system of organisms: Proposal for the domains of archaea, bacteria, and eucarya. Proc. Natl. Acad. Sci. USA 1990, 87, 4576-4579.

25. Rojas, M.R.; Zerbini, F.M.; Allison, R.F.; Robert L. Gilbertson; Lucas, W.J. Capsid protein and helper component-proteinase function as potyvirus cell-to-cell movement proteins. Virology 1997, 237, 283-295.

26. Chare, E.R. Selection pressures in the capsid genes of plant RNA viruses reflect mode of transmission. J. Gen. Virol. 2004, 85, 3149-3157.

27. Krupovic, M.; Ravantti, J.J.; Bamford, D.H. Geminiviruses: A tale of a plasmid becoming a virus. BMC Evol. Biol. 2009, 9, 112.

28. Martin, D.P.; Biagini, P.; Lefeuvre, P.; Golden, M.; Roumagnac, P.; Varsani, A. Recombination in eukaryotic single stranded DNA viruses. Viruses 2011, 3, 1699-1738.

29. Rosario, K.; Duffy, S.; Breitbart, M. A field guide to eukaryotic circular single-stranded DNA viruses: Insights gained from metagenomics. Arch. Virol. 2012, 157, 1851-1871.

30. Varsani, A.; Shepherd, D.N.; Dent, K.; Monjane, A.L.; Rybicki, E.P.; Martin, D.P. A highly divergent south african geminivirus species illuminates the ancient evolutionary history of this family. Virol. J. 2009, 6, 36.

31. Gutierrez, C. Geminivirus DNA replication. Cell. Mol. Life Sci. 1999, 56, 313-329.

32. Fuglsang, A. Estimating the "effective number of codons": The Wright way of determining codon homozygosity leads to superior estimates. Genetics 2005, 172, 1301-1307.

33. Adams, M.J.; Antoniw, J.F. Codon usage bias amongst plant viruses. Arch. Virol. 2004, 149, 113-135.

34. Jiang, Y.; Deng, F.; Wang, H.; Hu, Z. An extensive analysis on the global codon usage pattern of baculoviruses. Arch. Virol. 2008, 153, 2273-2282.

35. Liu, X.; Wu, C.; Chen, A.Y.H. Codon usage bias and recombination events for neuraminidase and hemagglutinin genes in chinese isolates of influenza a virus subtype h9n2. Arch. Virol. 2010, 155, 685-693.

36. Fu, M. Codon usage bias in herpesvirus. Arch. Virol. 2010, 155, 391-396.

37. Wang, M.; Zhang, J.; Zhou, J.-H.; Chen, H.-T.; Ma, L.-N.; Ding, Y.-Z.; Liu, W.-Q.; Liu, Y.-S. Analysis of codon usage in bovine viral diarrhea virus. Arch. Virol. 2010, 156, 153-160.

38. Wang, M.; Liu, Y.-S.; Zhou, J.-H.; Chen, H.-T.; Ma, L.-N.; Ding, Y.-Z.; Liu, W.-Q.; Gu, Y.-X.; Zhang, J. Analysis of codon usage in newcastle disease virus. Virus Genes 2011, 42, 245-253.

39. Xu, X.-Z.; Liu, Q.-P.; Fan, L.-J.; Cui, X.-F.; Zhou, X.-P. Analysis of synonymous codon usage and evolution of begomoviruses. Journal of Zhejiang University SCIENCE B 2008, 9, 667-674.

40. Gray, S.M.; Banerjee, N. Mechanisms of arthropod transmission of plant and animal viruses. Microbiol. Mol. Biol. Rev. 1999, 63, 128-148.

41. Power, A.G. Insect transmission of plant viruses: A constraint on virus variability. Current Opinions in Plant Biology 2000, 3.

42. Andret-Link, P.; Fuchs, M. Transmission specificity of plant viruses by vectors. Journal of Plant Pathology 2005, 87, 153-165.

43. Sanjuan, R.; Agudelo-Romero, P.; Elena, S.F. Upper-limit mutation rate estimation for a plant RNA virus. Biol. Lett. 2009, 5, 394-396. 
44. Gibbs, A.J.; Ohshima, K.; Phillips, M.J.; Gibbs, M.J. The prehistory of potyviruses: Their initial radiation was during the dawn of agriculture. PLOS ONE 2008, 3, e2523.

45. Pagan, I.; Holmes, E.C. Long-term evolution of the luteoviridae: Time scale and mode of virus speciation. J. Virol. 2010, 84, 6177-6187.

46. Duffy, S.; Holmes, E.C. Phylogenetic evidence for rapid rates of molecular evolution in the single-stranded DNA begomovirus tomato yellow leaf curl virus. J. Virol. 2008, 82, 957-965.

47. Duffy, S.; Holmes, E.C. Validation of high rates of nucleotide substitution in geminiviruses: Phylogenetic evidence from east african cassava mosaic viruses. J. Gen. Virol. 2009, 90, 15391547.

48. Harkins, G.W.; Martin, D.P.; Duffy, S.; Monjane, A.L.; Shepherd, D.N.; Windram, O.P.; Owor, B.E.; Donaldson, L.; van Antwerpen, T.; Sayed, R.A., et al. Dating the origins of the maizeadapted strain of maize streak virus, msv-a. J. Gen. Virol. 2009, 90, 3066-3074.

49. Lefeuvre, P.; Harkins, G.W.; Lett, J.-M.; Briddon, R.W.; Chase, M.W.; Moury, B.; Martin, D.P. Evolutionary time-scale of the begomoviruses: Evidence from integrated sequences in the nicotiana genome. PLoS ONE 2011, 6, e19193.

50. Hofacker, I.L.; Stadler, P.F.; Stocsits, R.R. Conserved rna secondary structures in viral genomes: A survey. Bioinformatics 2004, 20, 1495-1499.

51. Hofacker, I.L.; Fekete, M.; Flamm, C.; Huynen, M.A.; Rauscher, S.; Stolorz, P.E.; Stadler, P.F. Automatic detection of conserved rna structure elements in complete rna virus genomes. Nucleic Acids Res. 1998, 26, 3825-3836.

52. Grantham, R.; Gautier, C.; Guoy, M.; Mercier, R.; Pave, A. Codon catalog usage and the genome hypothesis. Nucleic Acids Res. 1980, 8, r49-r62.

53. Frederico, L.A.; Kunkel, T.A.; Shaw, B.R. A sensitive genetic assay for the detection of cytosine deamination: Determination of rate constants and the activation energy. Biochemistry (Mosc.) 1990, 29, 2532-2537.

54. Liu, X.-S.; Zhang, Y.-G.; Fang, Y.-Z.; Wang, Y.-L. Patterns and influencing factor of synonymous codon usage in porcine circovirus. Virol. J. 2012, 9, 68.

55. Shen, C.K.; Ikoku, A.; Hearst, J.E. A specific DNA orientation in the filamentous bacteriophage $\mathrm{fd}$ as probed by psoralen crosslinking and electron microscopy. J. Mol. Biol. 1979, 127, 163-175.

56. Incardona, N.L.; Prescott, B.; Sargent, D.; Lamba, O.P.; Thomas, G.J. Phage phi x174 probed by laser raman spectroscopy: Evidence for capsid-imposed constraint on DNA secondary structure. Biochemistry (Mosc.) 1987, 26, 1532-1538.

57. Benevides, J.M.; Stow, P.L.; Ilag, L.L.; Incardona, N.L.; Thomas, G.J. Differences in secondary structure between packaged and unpackaged single-stranded DNA of bacteriophage phi x174 determined by raman spectroscopy: A model for phi x174 DNA packaging. Biochemistry (Mosc.) 1991, 30, 4855-4863.

58. Welsh, L.C.; Marvin, D.A.; Perham, R.N. Analysis of x-ray diffraction from fibres of pf1 inovirus (filamentous bacteriophage) shows that the DNA in the virion is not highly ordered. $J$. Mol. Biol. 1998, 284, 1265-1271.

59. Wen, Z.Q.; Armstrong, A.; Thomas Jr, G.J. Demonstration by ultraviolet resonance raman spectroscopy of differences in DNA organization and interactions in filamentous viruses pf1 and fd. Biochemistry (Mosc.) 1999, 38, 3148-3156. 
60. Tsuboi, M.; Tsunoda, M.; Overman, S.A.; Benevides, J.M.; Thomas, G.J. A structural model for the single-stranded DNA genome of filamentous bacteriophage pf1. Biochemistry (Mosc.) 2010, 49, 1737-1743.

61. Sun, Y.; Chen, A.Y.; Cheng, F.; Guan, W.; Johnson, F.B.; Qiu, J. Molecular characterization of infectious clones of the minute virus of canines reveals unique features of bocaviruses. J. Virol. 2009, 83, 3956-3967.

62. Mol, C.D.; Parikh, S.S.; Putname, C.D.; Lo, T.P.; Tainer, J.A. DNA repair machanisms for the recognition and removal of damaged DNA bases. Annual Review of Biophysics and Biomolecular Structures 1999, 28, 101-128.

63. McClelland, M. Selection against dam methylation sites in the genomes of DNA of enterobacteriophages. J. Mol. Evol. 1985, 21, 317-322.

64. Bishop, K.N.; Holmes, R.K.; Sheehy, A.M.; Davidson, N.O.; Cho, S.-J.; Malim, M.H. Cytidine deamination of retroviral DNA by diverse apobec proteins. Curr. Biol. 2004, 14, 1392-1396.

65. Hadfield, J.; Thomas, J.E.; Schwinghamer, M.W.; Kraberger, S.; Stainton, D.; Dayaram, A.; Parry, J.N.; Pande, D.; Martin, D.P.; Varsani, A. Molecular characterisation of dicot-infecting mastreviruses from Australia. Virus Res. 2012, 166, 13-22.

66. Puigbò, P.; Bravo, I.G.; Garcia-Vallve, S. Caical: A combined set of tools to assess codon usage adaptation. Biol. Direct 2008, 3, 38.

(C) 2013 by the authors; licensee MDPI, Basel, Switzerland. This article is an open access article distributed under the terms and conditions of the Creative Commons Attribution license (http://creativecommons.org/licenses/by/3.0/). 